\title{
CFD-based numerical simulation of cyclone separator for separating stigmas from petals of saffron
}

\author{
Javad Nemati, Babak Beheshti, Ali Mohammad Borghei \\ Department of Biosystems Engineering, Science and Research Branch, Islamic Azad University, Tehran, Iran
}

\begin{abstract}
This study modelled numerically the flow of a fluid (air) and solid particles (saffron flower) inside a cyclone using the finite volume method in ANSYS Fluent. The continuous phase was simulated under steady state condition, as initial condition, using the Reynolds stress model for turbulence at three constant inlet air velocities of $1.5 \mathrm{~m} / \mathrm{s}, 2.5 \mathrm{~m} / \mathrm{s}$, and $3.5 \mathrm{~m} / \mathrm{s}$ over the inlet section. One-way coupling was assumed to govern all numerical analyses. The fluid phase and particles were treated as continuous medium (within a Eulerian framework) and discrete phase (within a Lagrangian framework), respectively. The equations governing the gas phase included the compressible Navier-Stokes equation and the conservation of mass equation. The discrete phase equations included the equations of motion for three different particles, including petals, stigmas, and anthers. According to the numerical results, the cyclone separation efficiency was calculated, and the static pressure and velocity contours were plotted. The results showed that the computational fluid dynamics-based simulation can provide an accurate demonstration of the behaviour of the fluid-solid phase. Accordingly, it can be used to predict the efficiency of stigma separation from petals of saffron using the airflow in the cyclone. According to the results, the highest cyclonic separation efficiency of $89 \%$ was achieved at an inlet air velocity of $3.5 \mathrm{~m} / \mathrm{s}$, which was very close to the experimental data.
\end{abstract}

\section{Introduction}

Cyclones are widely used in different industries to control air pollution and separate solid particles from an airflow. The simple

\footnotetext{
Correspondence: Babak Beheshti, Department of Biosystems Engineering, Science and Research Branch, Islamic Azad University, Tehran, Iran.

E-mail: beheshti-b@srbiau.ac.ir

Key words: Separation method; cyclone efficiency; particle density.

Received for publication: 16 June 2020.

Accepted for publication: 28 August 2020

(C) Copyright: the Author(s), 2020

Licensee PAGEPress, Italy

Journal of Agricultural Engineering 2020; LI:1091

doi:10.4081/jae.2020.1091

This article is distributed under the terms of the Creative Commons Attribution Noncommercial License (by-nc 4.0) which permits any noncommercial use, distribution, and reproduction in any medium, provided the original author(s) and source are credited.
}

fabrication process, low operating costs, and flexibility under difficult conditions have turned cyclones into one of the most important pieces of equipment for separating solid particles from an airflow (Zhao et al., 2006). Despite the simple geometry of cyclones, various studies indicated the complexity of rotational flows formed in this unit (Dias et al., 2008).

The review of the history of cyclones shows that the first cyclonic separator was patented in 1885 by Finch (Slack et al., 2000). Stairmand and Lapple conducted pioneering work in this area and proposed practical design formulas, which are still used for modelling most industrial cyclones. Attempts made by Stairmand and Lapple from 1950 to 1990 resulted in the design of classic cyclones and the development of equations that revealed the higher efficiency of classic cyclones compared to other cyclones for different particle diameters and inlet velocities (Altmeyer et al., 2004).

Boysan et al. (1982) published the results of the first gas cyclone modelling. They used the algebraic stress model to calculate six components of Reynolds stress (Ma et al., 2000).

A literature review showed that there are many studies on the simulation of cyclones. However, Boysan et al. (1982) were among the pioneers who used the computational fluid dynamics (CFD) to simulate cyclonic separators using Reynolds stress model (RSM). Slack et al. (2000) applied the RSM and large eddy simulation (LES) for cyclone modelling on a 3D unstructured grid and compared the simulation results with those obtained from the Doppler fuzzy speedometer (Wang et al., 2006).

Wang et al. (2006) developed analytical equations for the number of effective turns of particles in vortices inside a cyclone and investigated the factors affecting the cyclone pressure drop. Their results showed an increase in pressure drop, when the particle diameter and spray rate increased. Chu et al. (2009) investigated the CFD methods for Lagrangian particle tracking in dense medium cyclones (DMCs) (Umucu, 2015). Vince et al. (2014) optimized DMCs to inject an adequate number of coarser particles into the cyclone. They found that longer and wider cyclones offer higher efficiency and higher capacity, respectively.

Xiong et al. (2014) analysed six cyclones with different layouts for the outlet cylinder (vortex finder) both numerically and experimentally. According to their results, the cyclones with a conical vortex finder were more efficient than those with a cylindrical outlet. Moreover, a lower pressure drop (27\%) and higher efficiency (by $9 \%$ ) were observed in the cyclone with a slotted outlet section compared to that with a conical outlet.

Hsiao et al. (2015) investigated the effects of inlet and outlet sections, and the geometry of the conical section of the cyclone. According to their results, the performance of the cyclone can be improved by decreasing the length and increasing the width of the conical section. In order to achieve greater efficiency, the inlet and outlet sections should be made, respectively, larger and smaller to the extent that the pressure drop does not increase. The lack of a conical section or its replacement with a cylindrical section caused a decrease in cyclone efficiency. 
Song et al. (2016) investigated the length of the vortex formed in the cyclone both numerically and experimentally. The results showed a decrease in the rotational flow in the axial direction towards the cyclone bottom. In addition, the tangential and axial velocities gradually reduced in the same direction. Moreover, the kinetic energy of the flow in the cyclone was applied both radially and axially, and the energy dissipation caused a reduction in the flow intensity inside the cyclone. In this regard, an equation was proposed for the natural vortex length based on the geometry and intensity of the rotational flow (Li et al., 2016).

Jairo et al. performed a computational fluid dynamics assessment of the effect of different opening configurations on the thermal environment of a facility for coffee wet processing and compared the results (Jairo et al., 2019).

The literature indicates the importance and application of numerical modelling of cyclones for the separation of solid particles from gas flows. For the first time, this study investigates the numerical modelling of a cyclone to separate saffron stigma from its petals. The objectives and innovative features of this study are summarized as follows: i) numerical modelling of a cyclonic separator to separate saffron stigma from its petals; ii) the investigation of velocity and pressure fields inside the cyclone at different inlet air velocities; iii) the calculation of the cyclone efficiency in the separation of saffron stigma from its petals.

\section{Materials and methods}

Today, separators are used to separate agricultural products or product components, and/or remove impurities. A cyclonic separator is a piece of equipment for the collection of the finished product in the processing operation. In this separator, air and materials (saffron flowers) enter the cyclone tangentially from the top, where the pressure drops and the air creates a vortex in the centre of the cyclone chamber. The collision of materials (saffron flowers) against the cyclone wall causes the separation of its components and the accumulation of an air vortex and lighter materials (saffron stigma) at the centre, leaving the cyclone from the upper outlet. The heavier materials (petals) slip on the cyclone wall and leave it from the lower outlet (Figure 1A).

\section{The geometry of the cyclone and the experimental setup}

The geometry of the cyclone under investigation is presented in Figure 1B. This study was aimed at investigating the separation of flower components and the separation efficiency, therefore, also the conical section of the cyclone was considered. Therefore, it was assumed that the separation process was completed, when the particles reached the outlet. A 2D2D cyclone was used in this study and Eqs. (1-7) were used to obtain the dimensions of different parts of the cyclone. In these Eqs., $\mathrm{D}_{\mathrm{c}}$ represents the cyclone diameter. Table 1 shows the geometrical dimensions used in this study.

$\mathrm{B}_{\mathrm{c}}=\mathrm{D}_{\mathrm{c}} / 4$

$\mathrm{D}_{\mathrm{e}}=\mathrm{D}_{\mathrm{c}} / 2$

$\mathrm{H}_{\mathrm{c}}=\mathrm{D}_{\mathrm{c}} / 2$

$\mathrm{J}_{\mathrm{c}}=\mathrm{D}_{\mathrm{c}} / 4$

$\mathrm{L}_{\mathrm{c}}=2 * \mathrm{D}_{\mathrm{c}}$

$\mathrm{S}_{\mathrm{c}}=\mathrm{D}_{\mathrm{c}} / 8$

$\mathrm{Z}_{\mathrm{c}}=2 * \mathrm{D}_{\mathrm{c}}$

To validate the numerical results of our cyclone simulation (cyclone efficiency), two prototypes were designed and developed. The cyclone separation efficiency was measured by performing various tests. Figure 2 shows the designed device and its components for saffron stigma separation. Figure $3 \mathrm{~A}$ also shows a prototype of the saffron stigma separator.

Table 1. Specifications of the cyclone used in the calculations $(\mathrm{cm})$.

\begin{tabular}{ccccccccc}
$\mathrm{B}_{\mathrm{c}}(\mathrm{cm})$ & $\mathrm{H}_{\mathrm{c}}(\mathrm{cm})$ & $\mathrm{J}_{\mathrm{c}}(\mathrm{cm})$ & $\mathrm{S}_{\mathrm{c}}(\mathrm{cm})$ & $\mathrm{Z}_{\mathrm{c}}(\mathrm{cm})$ & $\mathrm{L}_{\mathrm{c}}(\mathrm{cm})$ & $\mathrm{D}_{\mathrm{e}}(\mathrm{cm})$ & $\mathrm{D}_{\mathrm{c}}(\mathrm{cm})$ \\
5 & 10 & 5 & 2.5 & 40 & 40 & 10 & 20 \\
\hline
\end{tabular}

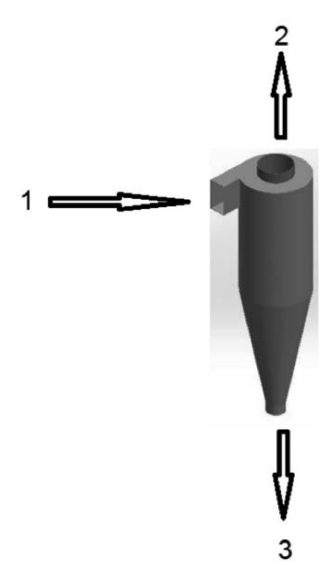

Figure 1. A) The schematic structure of the cyclone: 1. Cyclone inlet (air and solid), 2. Air and light materials outlet, 3. Heavy fraction outlet. B) The cyclone geometry used in this study.

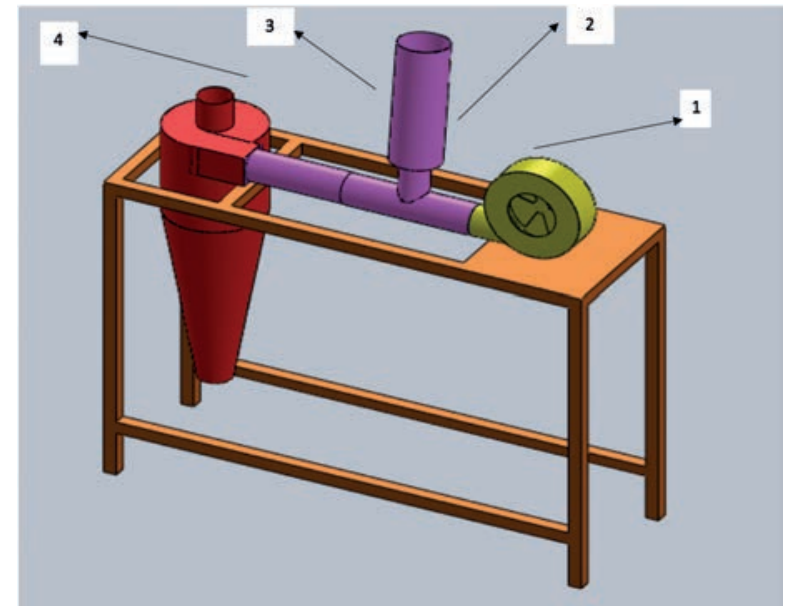

Figure 2. Design of saffron stigma separation machine in excellent work (Excellent Work?) software (1. Chassis; 2. Fan; 3. Tank; 4. Cyclone separator). 


\section{Meshing geometry}

In this study, a relatively unstructured fine grid with 1,033,042 prismatic and tetrahedral cells was used for numerical simulation. Figure 3B shows the grid used in numerical simulations, which was created in Gambit. The created mesh was capable of capturing the details of the flow field well. To reduce computational costs, instead of using the LES model, the steady-state simulation of the continuous phase was started with the RSM turbulence model using the cyclone inlet values as the initial condition.

The inlet air velocities of $1.5,2.5$, and $3.5 \mathrm{~m} / \mathrm{s}$ were used based on the results from the calculation and measurement of the velocities of saffron flower components.

\section{Governing equations}

The Euler-Lagrange method was used in ANSYS Fluent (ANSYS Fluent User's Guide, Release 17.2. ANSYS Inc., 2016) to simulate the flow of solid particles in the air. The airflow was solved as the continuous phase (within the Eulerian framework); whereas, the dispersed phase was calculated as the distribution of a large number of particles in the continuous phase (within the Lagrangian framework). In the Eulerian-Lagrangian approach, both equations governing the continuous and discrete phases were solved. In this approach, the continuous phase equations were coupled with discrete phase equations via the mass, momentum, and energy source terms.

\section{Governing equations in the continuous phase (air)}

The governing equations in the continuous phase (air) and the time-averaged Navier-Stokes equations, including the continuous and momentum balance equations for a viscous incompressible fluid, are represented by Eqs. (8) and (9), respectively:

$$
\frac{\partial \bar{u}_{i}}{\partial x_{i}}=0
$$

$$
\frac{\partial \bar{u}_{i}}{\partial t}+\bar{u}_{j} \frac{\partial \bar{u}_{i}}{\partial x_{j}}=-\frac{1}{\rho} \frac{\partial \bar{P}}{\partial x_{i}}+v \frac{\partial^{2} \bar{u}_{i}}{\partial x_{j} \partial x_{j}}-\frac{\partial R_{i j}}{\partial x_{j}}
$$

where $R_{i j}=\overline{u_{\imath}^{\prime} u_{\jmath}^{\prime}}$ represents the Reynolds stress tensor, and $u_{i}^{\prime}=u_{i}-\bar{u}_{i}$.

Various turbulence models are available to predict the turbulent rotational flow inside the cyclone, among which the RSM is the most accurate predictor of velocity, pressure, rotational (turbulent) flow, and efficiency of the cyclone (Shukla et al., 2013; Xu et al., 2016; Prabhansu et al., 2017). In this simulation, the RSM was used to predict the fluid (air) behaviour. In the RSM, the transfer equations were solved for every Reynolds stress term, resulting in an accurate prediction of the rotational flow pattern, velocity, and pressure. The transfer equation of the RSM was as follows:

$$
\begin{aligned}
& \frac{\partial}{\partial t}\left(\rho \overline{u_{\imath} u_{\jmath}^{\prime}}\right)+\frac{\partial}{\partial x_{k}}\left(\rho u_{k} \overline{\overline{u_{l} u_{j}}}\right)=\frac{\partial}{\partial x_{k}}\left(\frac{\mu_{t}}{\sigma_{k}} \frac{\partial \overline{u_{i} u_{j}}}{\partial x_{k}}\right)-\rho\left(\overline{\overline{u_{\imath} u_{k}^{\prime}}} \frac{\partial u_{j}}{\partial x_{k}}+\overline{\overline{u_{\jmath} u_{k}^{\prime}}} \frac{\partial u_{i}}{\partial x_{k}}\right)+
\end{aligned}
$$

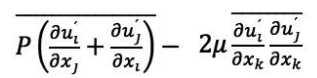

$\mu_{t}$ in Eq. (10) is calculated from Eq. (11):

$$
\mu_{t}=\rho C_{\mu} \frac{k^{2}}{\varepsilon}
$$

$\mathrm{k}$ and $\varepsilon$ in Eq. (11) were calculated from Eqs. (12) and (13), respectively:

$$
\frac{\partial}{\partial t}(\rho k)+\frac{\partial}{\partial x_{i}}\left(\rho k u_{i}\right)=\frac{\partial}{\partial x_{j}}\left[\left(\mu+\frac{\mu_{t}}{\sigma_{k}}\right) \frac{\partial k}{\partial x_{j}}\right]+\frac{1}{2}\left(P_{i i}+G_{i i}\right)-\rho \varepsilon\left(1+2 M_{t}^{2}\right)
$$

$$
\frac{\partial}{\partial t}(\rho \varepsilon)+\frac{\partial}{\partial x_{i}}\left(\rho \varepsilon u_{i}\right)=\frac{\partial}{\partial x_{j}}\left[\left(\mu+\frac{\mu_{t}}{\sigma_{\varepsilon}}\right) \frac{\partial \varepsilon}{\partial x_{j}}\right]+C_{\varepsilon 1} \frac{1}{2}\left(P_{i i}+C_{\varepsilon 3} G_{i i}\right) \frac{\varepsilon}{k}-C_{\varepsilon 2} \rho\left(\frac{\varepsilon^{2}}{k}\right)
$$

The constant values in the RSM were (Shukla et al., 2013).

\section{Dispersed phase model}

The flow of solid particles in the continuous phase was investigated by tracking the particles in the continuous flow. The drag force, acting on the particles, was investigated separately. The interactions between the particles and the effects of solid particles on the gas phase were not considered. The one-way coupling was assumed in the calculations. Subsequently, the equations governing the continuous phase were solved first, then also the equations governing the discrete phase were solved. As a result, the momentum equation governed the solid particles, where particle velocity variations were calculated based on Newton's second law of
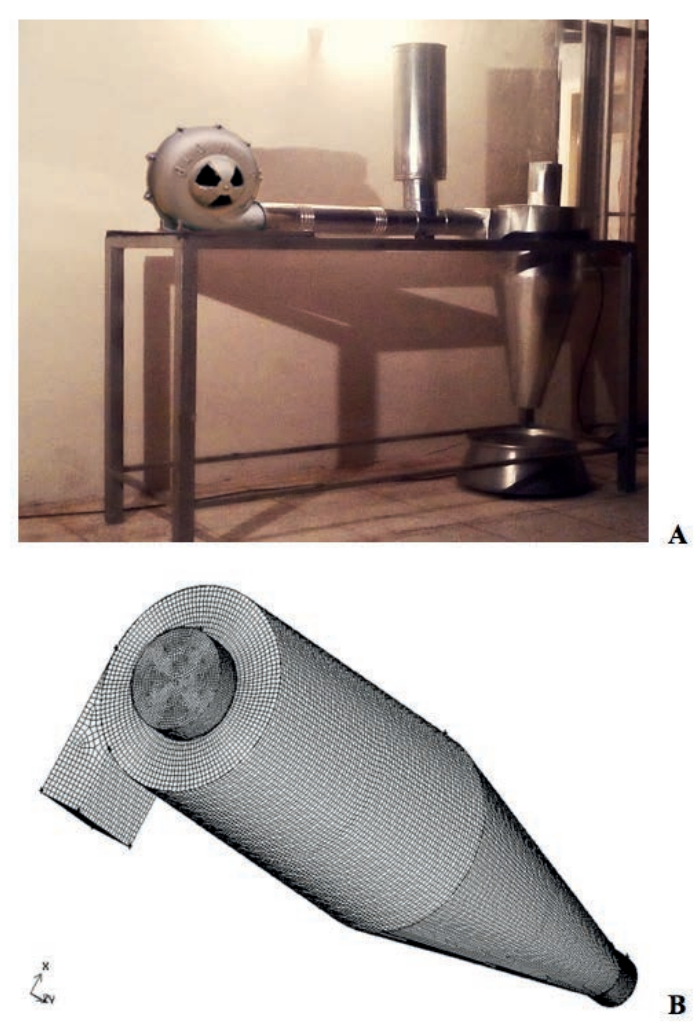

Figure 3. A) Prototype of saffron stigma separator. B) Computational grid used in numerical simulations. 
motion. The behaviour of particles is described by Eqs. 14 and 15:

$$
\begin{aligned}
& \frac{\partial u_{p i}}{\partial t}=f_{D}+\frac{g_{i}\left(\rho_{p}-\rho\right)}{\rho_{p}} \\
& \frac{\partial x_{p i}}{\partial t}=u_{p i}
\end{aligned}
$$

$f_{D}$ in Eq. (14) is calculated by Eq. (16).

$$
f_{D}=\frac{18 \mu}{\rho_{p} d_{p}^{2}} \frac{C_{D} R e_{p}}{24}\left(u_{i-} u_{p i}\right)
$$

In this paper, it was assumed that the particles were spherical with a smooth surface. Therefore, $R e_{p}$ and $R_{D}$ were calculated by Eqs. 17 and 18, respectively (Zhao et al., 2006).

$$
\begin{aligned}
& R e_{p}=\frac{\rho_{p} d_{p}\left|u-u_{p}\right|}{\mu} \\
& C_{D}=\frac{24\left(1+0.15 R e^{0.687}\right)}{R e}
\end{aligned}
$$

\section{Cyclone efficiency}

The separation efficiency of solid particles (here, saffron stigma) defined by Eq. (19) was considered the most important parameter to control the cyclone performance (Boysan et al., 1982):

$$
\eta=1-\frac{n_{\text {escaped }}}{n_{\text {tracked }}}
$$

\section{Boundary conditions and numerical model}

Air was used as the default fluid in this problem. The air density and dynamic viscosity in simulations were, respectively, 1.225 $\mathrm{kgm}^{-3}$ and $1.7894 \times 10^{-5} \mathrm{kgm}^{-1} \mathrm{~s}^{-1}$. Assuming the presence of impermeable cyclone walls and non-slip conditions, the atmospheric pressure was used as the boundary condition at output. A uniform velocity profile was assumed at the air inlet based on physical reality and the literature (Zhao et al., 2006; Dias et al., 2008; Wasilewski et al., 2020). Therefore, different boundary conditions in this simulation, namely velocity, turbulence intensity, and characteristic length of turbulence, were determined for different boundaries. An inlet turbulence intensity of 5\% was considered, and the hydraulic diameter was used as the longitudinal scale of turbulence. The cyclone was simulated at three inlet air velocities of $1.5 \mathrm{~m} / \mathrm{s}, 2.5 \mathrm{~m} / \mathrm{s}$, and $3.5 \mathrm{~m} / \mathrm{s}$. A homogeneous particle distribution was assumed at the inlet, and the particles were injected at the same velocity of the fluid. The boundary conditions for the particles were set to allow the reflection of the solid particles from the walls and their escape from the vortex finder. The SIMPLE design was used for pressure-velocity coupling and the first-order upwind method with greater stability was employed to discretize all equations. Moreover, a convergence criterion of $10^{-5}$ was considered for the remaining equations, as suggested by $\mathrm{Xu}$ et al. (2016). Three different particles, namely petals, stigmas, and anthers, were used in the numerical simulation. Table 2 presents the characteristics of the solid particles including mass, mean humidity, density, volume, total volume, and equivalent diameter. The density, volume, and equivalent diameter were calculated from Eqs. 20-22, respectively. The total volume was obtained by multiplying the particle volume by the number of particles (petals: 6, stigmas: 3, and anthers: 3). Equation 20 was used from Emadi and Saiedirad (2011).

$$
\begin{aligned}
& \rho_{b(\text { Petal })}=0.0001 M_{C}+0.0306 \\
& \rho_{b(\text { Anther })}=0.0007 M_{C}+0.1119 \\
& \rho_{b(\text { Stigma })}=-0.0009 M_{C}+0.1713
\end{aligned}
$$

Volume $=\mathrm{m} / \rho_{\mathrm{b}}$

$\mathrm{D}_{\mathrm{eq}}=(6 \times \text { Total volume } / \pi)^{1 / 3}$

Assuming that five saffron flowers enter the cyclone at each stage, $C_{\text {inlet,i }}$ and were calculated from Eqs. 23 and 24, respectively (Hinds, 1999). A inlet represented the inlet section area and was set at $0.1 \times 0.05 \mathrm{~m}^{2}$ in this study.

$$
\begin{aligned}
& C_{\text {inlet }, i}=\frac{5 m_{i}}{A_{\text {inlet }} D_{e q}} \\
& m_{\text {in jection }, i}=C_{\text {inlet }, i} A_{\text {inlet }} V_{\text {inlet }}
\end{aligned}
$$

Table 3 lists the inlet concentration $\left(\mathrm{C}_{\text {inlet }}\right)$ and the injection flow rate of particles $\left(m_{\text {in jection }}\right)$ at different inlet velocities.

The steady-state motion of particles was analysed. After defining the particle characteristics in Table 2, petals, stigmas, and anthers were injected separately at the cyclone inlet. After adjusting the discrete phase model (DPM) and these three injections, the flow, turbulence, and Reynolds stress equations were deactivated. Finally, the boundary conditions of the solid particles were applied at the boundaries before calculations.

\section{Results}

\section{Continuous phase}

Figure 4 presents the mean velocity (the modulus of the mean velocity vector) distribution at different inlet velocities at $\mathrm{z}=0.4 \mathrm{~m}$ and $z=0.6 \mathrm{~m}$ obtained from the numerical simulation of the steadystate equations for the continuous phase (air) with the RSM turbu-

Table 2. The characteristics of particles used in numerical simulation.

\begin{tabular}{lcccccc} 
Particle & $\mathrm{m}(\mathrm{g})$ & Mc $($ Moisture content, \%) & True density $\left(\mathrm{g} / \mathrm{cm}^{3}\right)$ & Volume $\left(\mathrm{mm}^{3}\right)$ & Total volume $\left(\mathrm{mm}^{3}\right)$ & Deq $(\mathrm{mm})$ \\
Petal & 0.045 & 47.81 & 0.728259 & 61.791 & 370.747 & 8.91 \\
Anther & 0.0264 & 35.83 & 1.406335 & 18.772 & 56.317 \\
\hline Stigma & 0.0045 & 33.83 & 0.99664 & 4.515 & 13.746 \\
\hline
\end{tabular}


lence model after convergence of the problem. As clearly seen, the velocity at $\mathrm{z}=0.4 \mathrm{~m}$, which was closer to the cyclone inlet, was greater than that at $\mathrm{z}=0.6 \mathrm{~m}$. Moreover, the velocity increased, when the inlet velocity increased on both cyclone sections. The high-velocity and low-velocity rotational areas can be seen near the walls and at the centre of the cyclone, respectively.

Figure 5 shows the velocity vectors inside the cyclone at different inlet velocities. As seen, an intense rotational flow was formed inside the cyclone. The velocity and rotational flow inside the cyclone increased, when the inlet velocity increased. These vectors clearly showed the air leaving from the upper outlet of the cyclone.

Figure 6A-C shows the velocity contours at the middle plane inside the cyclone at different velocities. The highest velocity was observed at the cyclone inlet, conversely the velocity was lower at the bottom of the cyclone. This result can be justified based on the law of conservation of angular momentum, according to which the centrifugal force is higher in the upper part of the cyclone leading to a larger angular momentum and consequently a higher velocity. The low- and high-velocity areas were, respectively, observed at the centre and near the walls of the cyclone.

Figure 6D-F shows the pressure contours in the cyclone middle plate at different velocities. As seen, the pressure distribution inside the cyclone was almost symmetric. The low- and high-pressure areas were at the centre and near the walls of the cyclone, respectively. This higher pressure was due to the rotational flow and a centrifugal force towards the cyclone walls. The formation of a low-pressure area and a relative vacuum at the top of the cyclone expelled the particles from the cyclone.
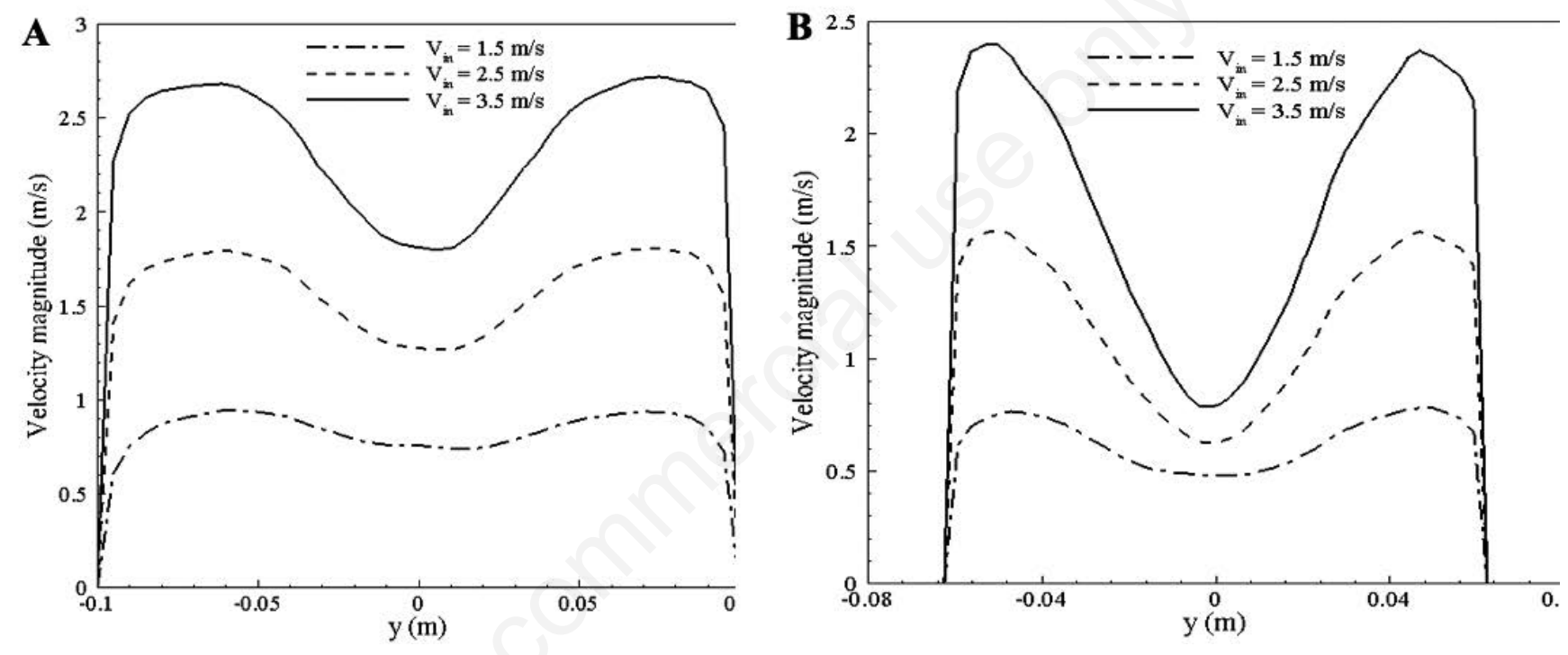

Figure 4. Mean velocity distribution at different inlet velocities: A) z=0.04 m, and B) $\mathrm{z}=0.6 \mathrm{~m}$.

Table 3. The inlet concentration and injection flow rate of solid particles.

\begin{tabular}{|c|c|c|c|}
\hline Air velocity $(\mathrm{m} / \mathrm{s})$ & Particle type & Inlet concentration $\left(\mathrm{g} / \mathrm{m}^{3}\right)$ & Injection flow rate $(\mathrm{kg} / \mathrm{s})$ \\
\hline \multirow[t]{3}{*}{1.5} & Petal & 30292.655 & 0.227195 \\
\hline & Anther & 16653.771 & 0.124903 \\
\hline & Stigma & 4564.587 & 0.034234 \\
\hline \multirow[t]{3}{*}{2.5} & Petal & 30292.655 & 0.378658 \\
\hline & Anther & 16653.771 & 0.208172 \\
\hline & Stigma & 4564.587 & 0.057057 \\
\hline \multirow[t]{3}{*}{3.5} & Petal & 30292.6545 & 0.530121 \\
\hline & Anther & 16653.771 & 0.291441 \\
\hline & Stigma & 4564.587 & 0.079880 \\
\hline
\end{tabular}

Table 4. Efficiency of stigma separation from petal in the cyclone under investigation at different inlet velocities.

\begin{tabular}{lccccc} 
Inlet velocity $(\mathrm{m} / \mathrm{s})$ & Tracked & Escaped & Trapped & Incomplete & Efficiency (\%) \\
1.5 & 200 & 34 & 2 & 164 & 17 \\
2.5 & 200 & 154 & 5 & 41 & 77 \\
\hline 3.5 & 200 & 178 & 10 & 12 & 89 \\
\hline
\end{tabular}




\section{Particle analysis}

Figure 7A-C shows the distribution contours for petals, anthers, and stigmas at different inlet velocities. As seen, the particles next to the wall had a lower velocity due to a non-slip condition on the wall, and the particles slightly away from the wall had a higher velocity.

Based on Figures 6D-F and 7A-F, the velocity of all three particles inside the cyclone increased, when the inlet velocity increased, therefore, the particles moved more towards the cyclone walls and travelled a longer distance. The diameter of petals was larger than that of stigmas and anthers. Therefore, they travelled a longer path inside the cyclone and finally stopped at the cyclone bottom, where the separation took place. Moreover, petals moved further towards the cyclone walls than stigmas and anthers and this could be attributed to the higher centrifugal force exerted on petals. The diameter of stigmas was smaller than that of two other particles. As a result, they travelled a shorter distance to leave the cyclone.

Table 4 shows the efficiency of stigma separation from petals in the cyclone for the injected particles at different inlet velocities. The highest separation efficiency was obtained at an inlet velocity of $3.5 \mathrm{~m} / \mathrm{s}$. The improved efficiency of stigma separation from petals, when the inlet velocity increased, could be related to an increase in the particle rotation at the inlet. Based on experimental data, the highest separation efficiency of $80 \%$ was obtained at a humidity of $20 \%$ (base) and a velocity of $3.5 \mathrm{~m} / \mathrm{s}$. Therefore, the numerical results were very close to the experimental data with an approximate difference of $11.25 \%$.

The main results are summarized below:

- The low- and high-velocity areas were, respectively, observed at the centre and near the walls of the cyclone, resulting in an increased centrifugal force acting on the larger petal particles which, in turn, facilitated the cyclonic separation.

- The pressure distribution inside the cyclone was almost symmetric. The generation of the centrifugal force resulted in the formation of high- and low-pressure areas respectively near the walls and at the cyclone centre. The formation of a low-pressure area and a relative vacuum at the top of the cyclone caused the expulsion of the particles.

- The velocity of all three particles inside the cyclone increased, when the inlet velocity increase, therefore, the particles moved more towards the cyclone walls and travelled a longer distance. These results can be seen in Figure 7.

- The diameter of petals was larger than that of stigmas and anthers, therefore, they travelled a longer distance inside the cyclone towards the cyclone bottom, where the separation took place.

- The diameter of stigmas was smaller than that of two other particles. As a result, they travelled a shorter distance to leave the cyclone.

- The rotational velocity inside the cyclone increased, when the inlet velocity increase, thus increasing, in turn, the cyclonic separation efficiency.

- The highest cyclonic separation efficiency of $89 \%$ was observed at an inlet velocity of $3.5 \mathrm{~m} / \mathrm{s}$.

\section{Conclusions}

The flow of air and saffron flower particles was simulated inside a cyclone using the CFD method. To this end, the Eulerian approach and DPM were applied to the airflow and particles, respectively. The RSM and three different particles, namely petals, stigmas, and anthers, were used in the simulation. The flow in the cyclone was simulated at three inlet air velocities of $1.5 \mathrm{~m} / \mathrm{s}, 2.5$ $\mathrm{m} / \mathrm{s}$, and $3.5 \mathrm{~m} / \mathrm{s}$. The reason for using air velocities of $1.5,2.5$, and $3.5 \mathrm{~m} / \mathrm{s}$ was to calculate and measure the speed limit (terminal

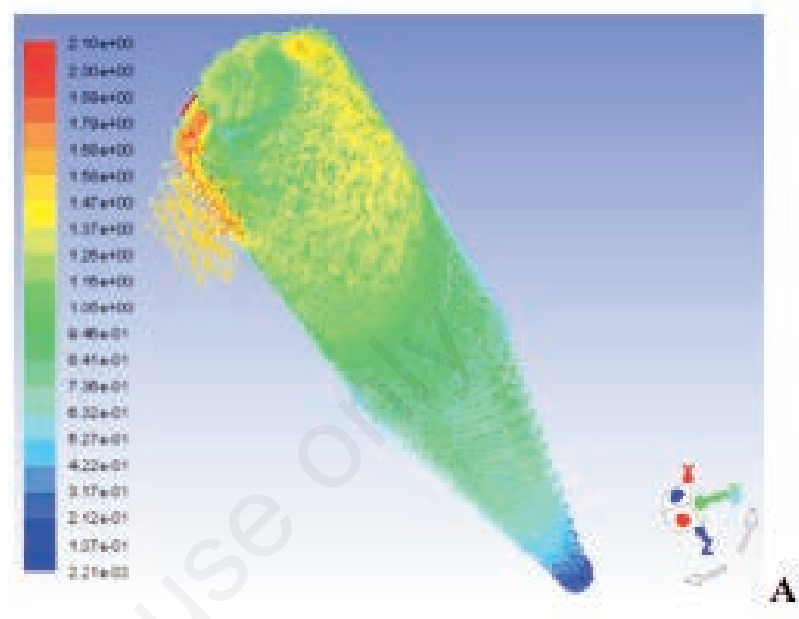

A
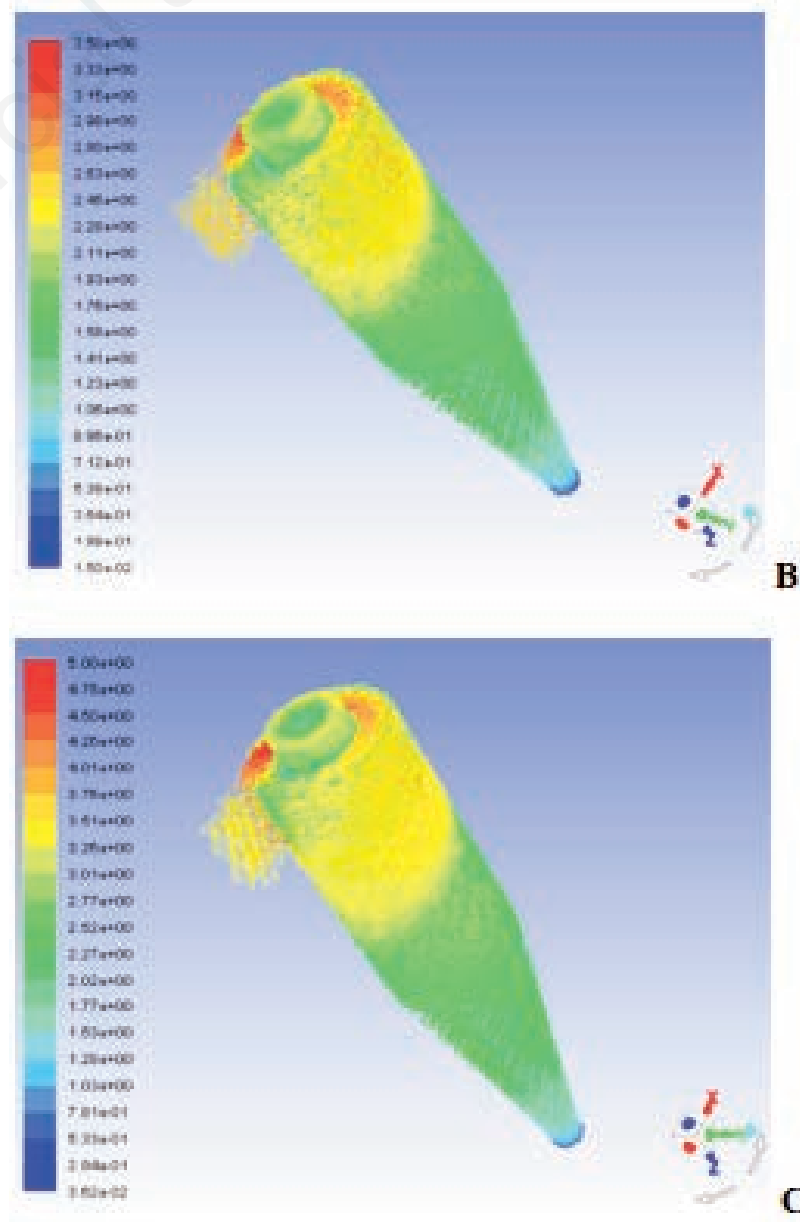

Figure 5. Mean velocity vectors $(\mathrm{m} / \mathrm{s})$ inside the cyclone at inlet velocities of A) $1.5 \mathrm{~m} / \mathrm{s}, \mathrm{B}) 2.5 \mathrm{~m} / \mathrm{s}$, and C) $3.5 \mathrm{~m} / \mathrm{s}$. 


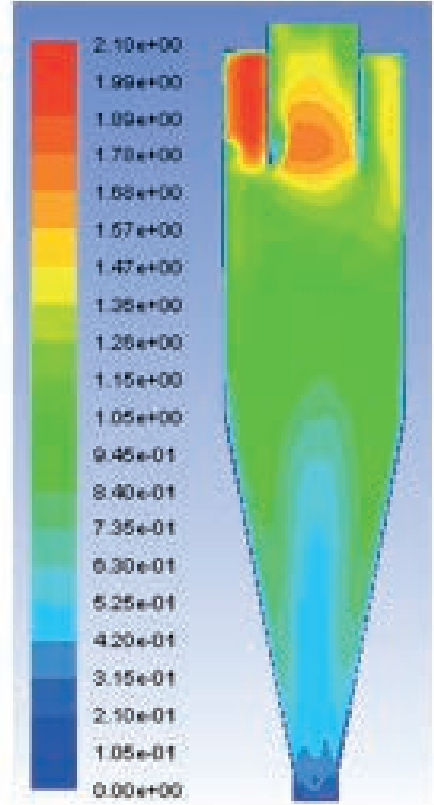

A

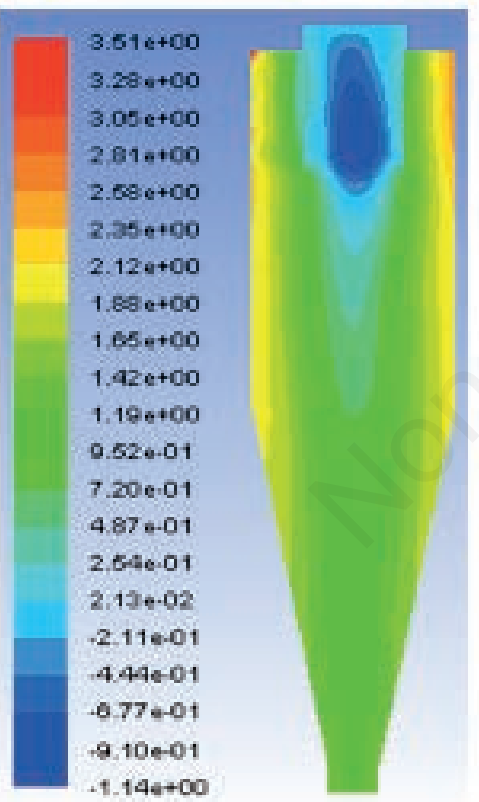

D

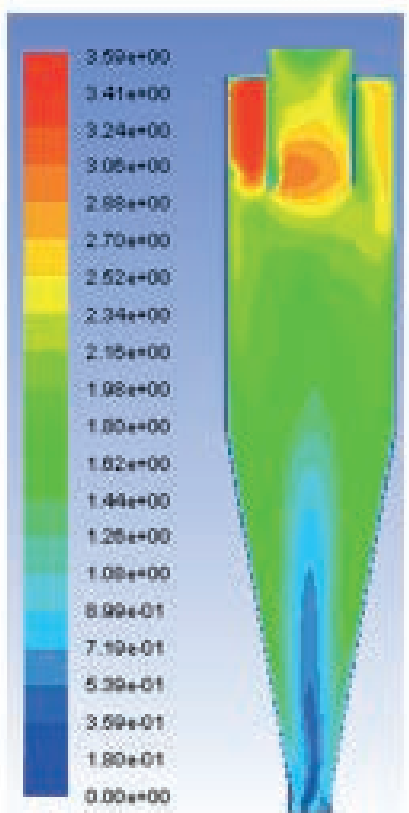

B

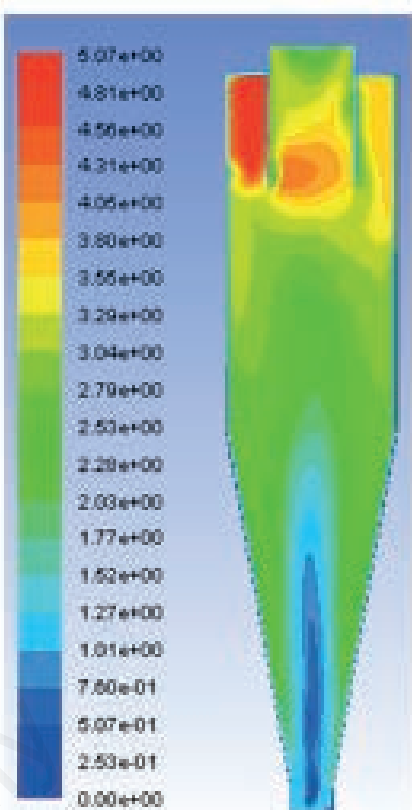

C 


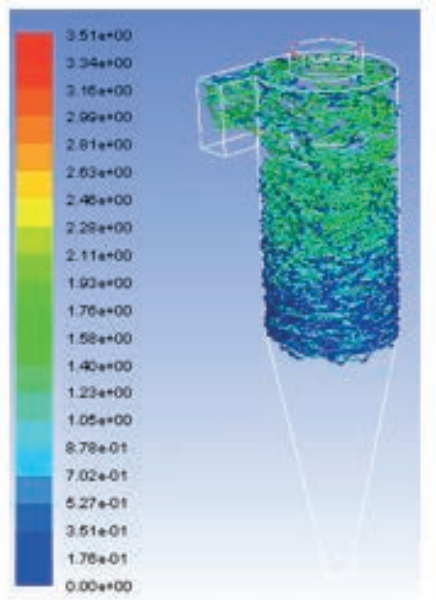

$\mathbf{A}$

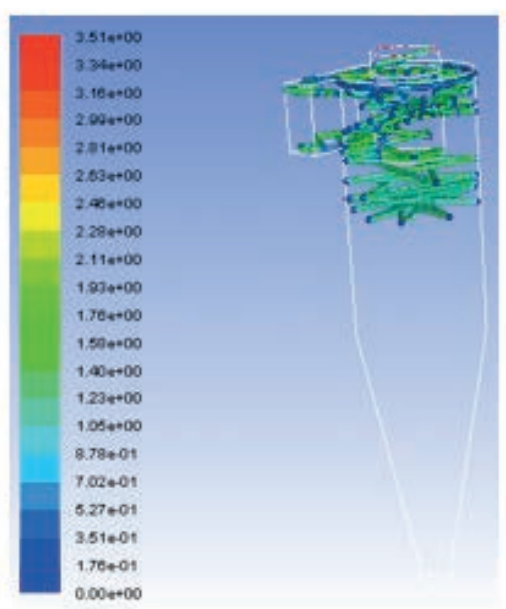

D

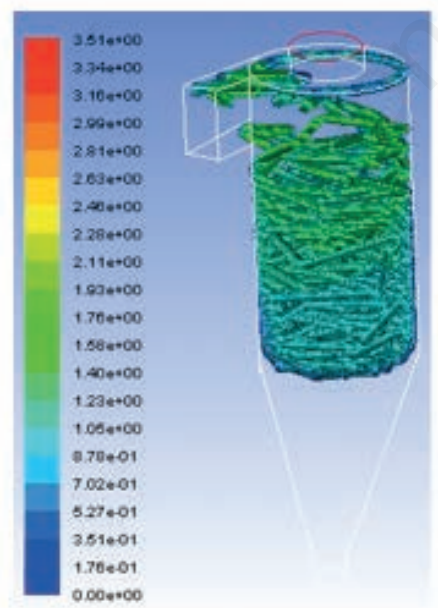

G

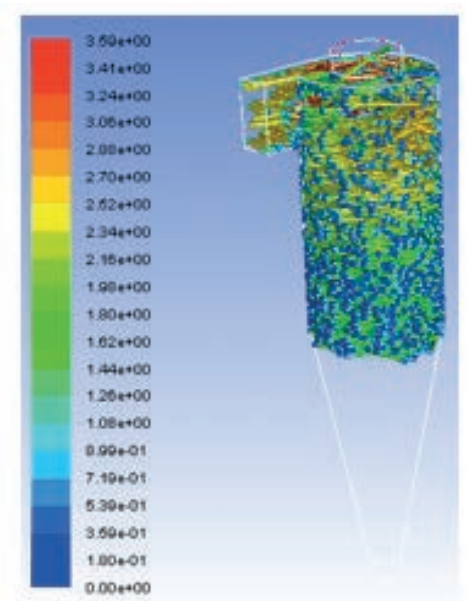

B

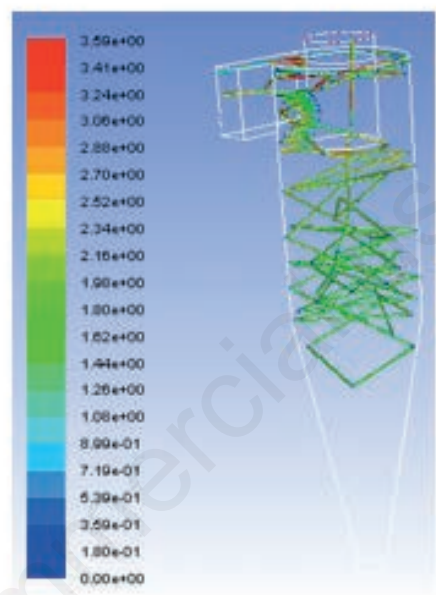

$\mathbf{E}$

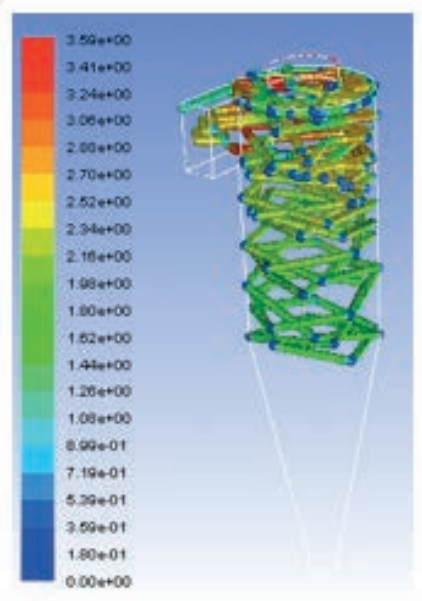

H

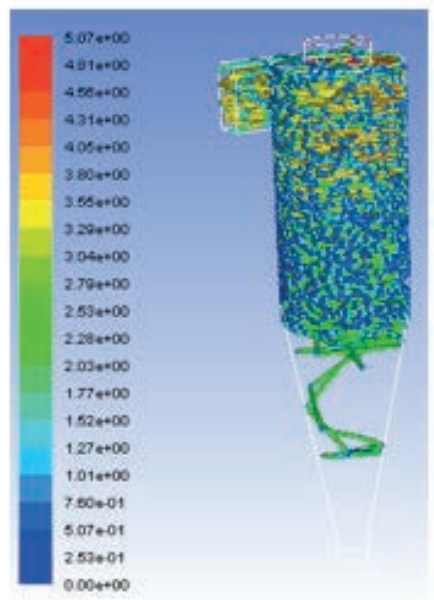

C

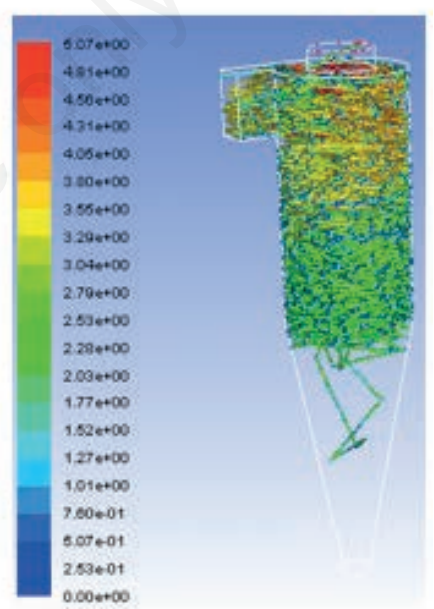

F

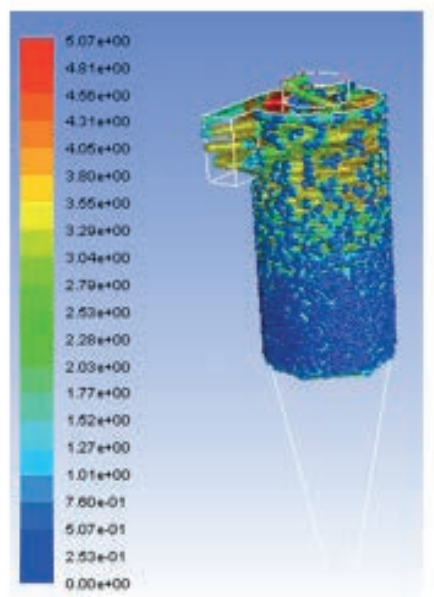

I

Figure 7. Particle distribution contours for petals at different inlet velocities: A) $1.5 \mathrm{~m} / \mathrm{s}$, B) $2.5 \mathrm{~m} / \mathrm{s}$, and C) $3.5 \mathrm{~m} / \mathrm{s}$; particle distribution contours for anthers at different inlet velocities: D) $1.5 \mathrm{~m} / \mathrm{s}, \mathrm{E}) 2.5 \mathrm{~m} / \mathrm{s}$, and F) $3.5 \mathrm{~m} / \mathrm{s}$; particle distribution contours for stigmas at different inlet velocities: G) $1.5 \mathrm{~m} / \mathrm{s}, \mathrm{H}) 2.5 \mathrm{~m} / \mathrm{s}$, and I) $3.5 \mathrm{~m} / \mathrm{s}$. 
velocity) of saffron flower components.

The results showed that the CFD method is capable of predicting the behaviour of saffron flower particles within the airflow in a cyclone. Therefore, this method can be used to predict the efficiency of stigma separation from petals using an airflow inside the cyclone.

This is the first study on the use of a cyclone to separate the saffron stigma from its petals, and its results can be used as an innovation to reduce the time and increase the efficiency of saffron separation. After simulating the cyclone, the prototype of the stigma separator was designed, developed, and evaluated. The experimental data from the device showed that the highest value was very close to the best simulation result using the CFD method. The best device performance was $89 \%$ at a velocity of $3.5 \mathrm{~m} / \mathrm{s}$.

\section{Nomenclature}

Cyclone diameter $(\mathrm{m})$

Cyclone efficiency

Dissipation rate of mixed flow $\left(\mathrm{m}^{2} / \mathrm{s}^{3}\right)$

Drag coefficient

Drag force for each particle's mass $\left(\mathrm{m} / \mathrm{s}^{2}\right)$

Dynamic viscosity of gas $(\mathrm{kg} / \mathrm{m} . \mathrm{s})$

Equivalent diameter of particles $(\mathrm{m})$

Gas density $\left(\mathrm{kg} / \mathrm{m}^{3}\right)$

Gas-phase velocity fluctuations $(\mathrm{m} / \mathrm{s})$ in i direction

$\mathrm{G}$-force in i direction

Inlet cross-section $\left(\mathrm{m}^{2}\right)$

Inlet velocity $(\mathrm{m} / \mathrm{s})$

Input particle concentration $\mathrm{i}\left(\mathrm{gr} / \mathrm{m}^{3}\right)$

Instantaneous gas velocity $(\mathrm{m} / \mathrm{s})$ in i direction

Kinematic viscosity of mixed flow $\left(\mathrm{m}^{2} / \mathrm{s}\right)$

Kinematic viscosity $\left(\mathrm{m}^{2} / \mathrm{s}\right)$

Kinetic energy of mixed flow $\left(\mathrm{m}^{2} / \mathrm{s}^{2}\right)$

Location $(\mathrm{m})$

Mass $(\mathrm{kg})$

Mass discharge of particle injection $(\mathrm{kg} / \mathrm{s})$

Mean gas velocity $(\mathrm{m} / \mathrm{s})$ in i direction

Mean humidity (\%)

Mean pressure $(\mathrm{Pa})$

Number of injected particles at cyclone inlet

Particle diameter $(\mathrm{m})$

Particle density $\left(\mathrm{kg} / \mathrm{m}^{3}\right)$

Particle velocity $(\mathrm{m} / \mathrm{s})$ in i direction

Reynolds stress tensor $\left(\mathrm{m}^{2} / \mathrm{s}^{2}\right)$

Reynolds number

Total number of particles leaving vortex finder

$\mathrm{D}_{\mathrm{c}}$
$\eta$
$\varepsilon$
$C_{D}$
$f_{D}$
$\mu$
$\mathrm{D}_{\mathrm{eq}}$
$\rho$
$u_{i}^{\prime}$
$g_{i}$
$\mathrm{~A}_{\text {inlet }}$
$\mathrm{V}_{\text {inlet }}$
$\mathrm{C}_{\text {inlet, }}$
$u_{i}$
$\mu_{t}$
$v$
$k$
$x_{i}$
$\mathrm{~m}$
$\dot{m}_{\text {in jection, } i}$
$\overline{u_{i}}$
$\mathrm{M}_{\mathrm{c}}$
$\bar{P}$
$n_{\text {tracked }}$
$d_{p}$
$\rho_{p}$
$u_{p i}$
$R_{i j}$
$R_{p}$
$n_{\text {escaped }}$

\section{References}

Altmeyer S., Mathieu V., Jullemier S., Contal P., Midoux N., Rode S., Leclerc J.P. 2004. Comparison of different models of cyclone prediction performance for various operating conditions using a general software. Chem. Eng. Process. 43: 51122.

Boysan F., Ayers W.H., Swithenbank J. 1982. A fundamental mathematical modeling approach to cyclone design. Trans. Inst. Chem. Eng. 16: 222-30.

Chu K.W., Wang B., Yu A.B., Vince A. 2009. Modeling the multi- phase flow in dense medium cyclones. $7^{\text {th }}$ International Conference on CFD in the Minerals and Process Industries, December 9-11, Australia.

Dias D., Mori M., Martignoni W. 2008. Study of different approaches for modeling cyclones using CFD. AIChE Annual Meeting.

Emadi B., Saiedirad M.H. 2011. Moisture-Dependent Physical Properties of Saffron Flower. J. Agr. Sci. Tech. 13: 387-98.

Hinds W.C. 1999. Aerosol technology, properties, behavior, and measurement of airborne particles, Second edition. John Wiley \& Sons, Inc., New York, USA.

Hsiao T.C., Huang S.H., Hsu C.W., Chen C.C. 2015. Effects of the geometric configuration on cyclone performance. J. Aerosol. Sci. 86: 1-12.

Jairo A., Velez-piedrahita S., Ciro-velasquez H.J., Largo-Avila E. 2019. Study of the effect of the geometry of a typical solar dryer for coffee using CFD. Rev. Ing. Univ. De Medellin 18:149-61.

Li X., Song J., Sun G., Jia M., Yan C., Yang Z., Wei Y. 2016. Experimental study on natural vortex length in a cyclone separator. Can. J. Chem. Eng. 94:2373-9.

Ma L., Ingham D.B., Wen X. 2000. Numerical modeling of the fluid and particle penetration through small sampling cyclones. J. Aerosol. Sci. 31: 1097-119.

Prabhansu R.S., Ganguli S., Chandra P., Karmakar M.K., Chatterjee P.K. 2017. Numerical analysis of gas-solid behavior in a cyclone separator for circulating fluidized bed system. J. Appl. Fluid. Mech. 10: 1167-76.

Shukla S.K., Shukla P., Ghosh P. 2013. The effect of modeling of velocity fluctuations on prediction of collection efficiency of cyclone separators. Appl. Math. Model 37: 5774-89.

Slack M.D., Prasad R.O., Bakker A., Boysan F. 2000. Advances in cyclone modeling using unstructured grid. AIChE J. 78: 1098104.

Song C., Pei B., Jiang M., Wang B., Xu D., Chen Y. 2016. Numerical analysis of forces exerted on particles in cyclone separators. Powder Technol. 294:437-48.

Umucu, Y. 2015. Investigation of separation performance of dense medium cyclone using computer simulation. Physicochem. Probl. M. 51: 303-14.

Vince A., Barnett G.D., Barnett P.J. 2014. How to optimize design and operation of dense medium cyclones in coal preparation. Miner. Eng. 62: 55-65.

Wang L., Parnell C.B., Shaw B.W., Lacey R.E. 2006. A theoretical approach for predicting number of turns and cyclone pressure drop. Biol. Eng. Trans. 49: 491-503.

Wasilewski M., Brar L.S., Ligus G. 2020. Experimental and numerical investigation on the performance of square cyclones with different vortex finder configurations. Sep. Purif. Technol. 239: 116588.

Xiong Z., Ji Z., Wu X. 2014. Development of a cyclone separator with high efficiency and low pressure drop in axial inlet cyclones. J. Powder. Technol. 253: 644-9.

Xu W., Li Q., Wang J., Jin Y. 2016. Performance evaluation of a new cyclone separator-part II simulation results. Sep. Purif. Technol. 160: 112-6.

Zhao B., Su Y., Zhang J. 2006. Simulation of gas flow pattern and separation efficiency cyclone with conventional single and spiral double inlet configuration. Chem. Eng. Res. Des. 84: 115865 . 